\title{
Complete Amino Acid Sequence of Neutral Protease from Bacillus subtilis var. amylosacchariticus
}

\author{
Ryuji Kobayashi, Tadashi Yoshimoto and Daisuke Tsuru \\ School of Pharmaceutical Sciences, Nagasaki University, \\ Bunkyo-machi 1-14, Nagasaki 852, Japan
}

Received May 1, 1989

\begin{abstract}
The neutral protease of Bacillus subtilis var. amylosacchariticus was cleaved chemically or digested with proteolytic enzymes, and the resultant peptides were separated and purified by high performance liquid chromatography. The sequence analyses of these peptides by the manual Edman procedure established the complete amino acid sequence of the enzyme. The neutral protease consisted of 300 amino acid residues with Ala and Leu as its amino- and carboxyl-termini, respectively, and the molecular weight was calculated to be 32,633 . The sequence was found to be identical to that of $B$. subtilis IA72 neutral protease, which was deduced from nucleotide sequencing. Comparison of the sequence with those of other Bacillus proteases revealed that the putative active site amino acid residues, $\mathrm{Zn}$-binding ligands, and two Ca-binding sites were well conserved among them, as compared with those of thermolysin.
\end{abstract}

Several species of the genus Bacillus produce neutral and alkaline proteases (subtilisins) together with $\alpha$-amylases, and their enzymatic properties have been reported. ${ }^{\prime \prime}$ Among them, comprehensive studies have been done on protein chemical structures of subtilisins ${ }^{2 \sim 4}$ ) and thermolysin, a thermostable neutral $\mathrm{Zn}$ metallo protease. ${ }^{5 \sim 7 \text {, }}$

Bacillus subtilis var. amylosacchariticus ( $B$. amylosacchariticus) was isolated from soil by Fukumoto et al. $^{8)}$ as a producer of a saccharifying type $\alpha$-amylase which was distinguishable from the liquefying type $\alpha$-amylase produced by Bacillus subtilis amyloliquefaciens (B. amyloliquefaciens). ${ }^{9}$ 'The former strain produces neutral protease (BANP) and subtilisin Amylosacchariticus (subtilisin AM) besides saccharifying type $\alpha$-amylase. Some enzymatic and physicochemical properties of these two proteolytic enzymes have been characterized by Tsuru et al. ${ }^{1,10 \sim 13)}$ and the amino acid sequence of subtilisin AM was determined from the protein sequencing by Kurihara et $a l{ }^{14)}$ and from nucleotide sequencing of the gene by Yoshimoto et al. ${ }^{15)}$ On the other hand, $B$. subtilis neutral protease was characterized as a $\mathrm{Zn}$-metalloenzyme with high specific activity, ${ }^{16,17)}$ but the protein chemical properties of this enzyme remained to be clarified. Thus, we attempted to establish the amino acid sequence of the enzyme by protein sequencing. In this paper, we report the complete amino acid sequence of the neutral protease from $B$. subtilis var. amylosacchariticus and discuss its active site structure in comparison with that of thermolysin.

\section{Materials and Methods}

Materials. Cyanogen bromide (CNBr), trifluoroacetic acid (TFA), and other sequencing grade chemicals were obtained from Nakarai Chemicals, Kyoto. Lysyl endopeptidase from Achromobacter lyticus and phenylthiohydantoin $(\mathrm{PTH})$ amino acids were from Wako Pure Chemicals, Osaka. Staphylococcus aureus V8 protease and endoproteinase Asp-N from a Pseudomonas fragi mutant were from Miles Laboratories, U.S.A. and Boehringer Mannheim, Germany, respectively. TPCK-treated trypsin, $\alpha$-chymotrypsin, and thermolysin were from Sigma, U.S.A. Phenylisothiocyanate was from Pierce, U.S.A.

Abbreviations: BANP, neutral protease from Bacillus subtilis var. amylosacchariticus; HPLC, high performance liquid chromatography; subtilisin AM, subtilisin Amylosacchariticus; TFA, trifluoroacetic acid; TPCK, $N^{2}$-tosyl phenylalanyl chloromethyl ketone; PTH-, phenylthiohydantoin; PTC, phenylthiocarbamyl. 
HPLC grade acetonitrile and 2-propanol were from Kishida Chemicals, Osaka.

Purification of Bacillus subtilis amylosacchariticus neutral protease. The neutral protease of Bacillus subtilis amylosacchariticus (BANP) was purified by the method of Tsuru et $a l^{10 /}$ with some modifications. Forty grams of crude powder of the enzyme (Uyeda Chemical Industries Co.,) were suspended in $200 \mathrm{ml}$ of $10 \mathrm{~mm}$ Tris-maleate buffer, $\mathrm{pH} 6.4$, containing $5 \mathrm{~mm} \mathrm{Ca}$ acetate, and the filtrate was put on a column $(3 \times 40 \mathrm{~cm})$ of CM-Sephadex equilibrated with the same buffer. After the column was washed with $300 \mathrm{ml}$ of the buffer, the enzyme was eluted with an increasing linear gradient of $\mathrm{NaCl}$ concentration $(0$ to $0.6 \mathrm{M}$ ). The active fractions eluted at $0.2 \mathrm{M} \mathrm{NaCl}$ were combined and concentrated by ultrafiltration, and then dialyzed against $10 \mathrm{~mm}$ Tris- $\mathrm{HCl}$ buffer, $\mathrm{pH} 8.0$, with $2 \mathrm{~mm}$ $\mathrm{Ca}$ acetate. The dialyzate, $100 \mathrm{ml}$, was put onto a column $(3 \times 40 \mathrm{~cm})$ of DEAE-Sephadex equilibrated with the same buffer, and the enzyme adsorbed was eluted with an increasing linear gradient of $\mathrm{NaCl}$ concentration.

Active fractions were combined, concentrated, and dialyzed against $5 \mathrm{~mm}$ Tris- $\mathrm{HCl}$ buffer, $\mathrm{pH} 7.2$, containing $2 \mathrm{mM} \mathrm{Ca}$ acetate and then chromatographed on a column $(3 \times 20 \mathrm{~cm})$ of DEAE-cellulose equilibrated with the bufer. The enzyme was found in break-through fractions, but the specific activity had increased significantly. The fractions which showed the specific activity higher than 9,000 were combined and dialyzed against $2 \mathrm{~mm} \mathrm{Ca}$ acetate and lyophilized. The final preparation appeared homogeneous, as judged by amino terminal amino acid analysis but was further chromatographed by reverse phase HPLC using a TOSO system CCPM-8000, just before sequence analysis, to remove impurities which might come from its autolysis; the enzyme was denatured by dissolving in $70 \%$ formic acid and chromatographed on a Vydac C4 column $(5 \mu \mathrm{m}$, $300 \AA, 4.6 \times 250 \mathrm{~mm}$; Separations Group, CA, U.S.A.) using a solvent system of $0.075 \%$ TFA in water vs. acetonitrile-2-propanol $(3: 1)$ with $0.06 \%$ TFA. The denatured protein was eluted by an increasing gradient of organic solvent concentration at a flow rate of $1.0 \mathrm{ml} / \mathrm{min}$ at room temperature.

Assays of proteolytic activity and protein concentration. The proteolytic activity was assayed by the method described previously, ${ }^{10}$ and the protein concentration was estimated spectrophotometrically by assuming that $E_{1 \% 1 \mathrm{~cm}}$ at $280 \mathrm{~nm}$ is $13.8 .^{10}$ The specific activity was defined as the activity units per mg of protein.

Chemical or enzymatic digestion of $B A N P$, separation of the resultant peptides and their amino acid sequence analysis CNBr-cleavage. BANP $(10 \mathrm{mg})$ was incubated with CNBr ( $30 \mathrm{mg}$ ) in $4.0 \mathrm{ml}$ of $70 \%$ formic acid for $24 \mathrm{hr}$ at room temperature, diluted with water, and then lyophilized.

$S$. aureus V8 protease digestion. Digestion with $S$ : aureus
$\mathrm{V} 8$ protease was done at $37^{\circ} \mathrm{C}$ in $0.1 \mathrm{M}$ ammonium acetate $(\mathrm{pH} 4.0$ ) or in $0.1 \mathrm{M}$ phosphate buffer ( $\mathrm{pH} 7.9$ ). BANP, $1 \mathrm{mg}$, was dissolved in $45 \mu \mathrm{l}$ of the above buffer and the V8 protease was added at the enzyme-substrate ratio of $1: 50$ (wt/wt). After $21 \mathrm{hr}$ of incubation at $37^{\circ} \mathrm{C}$, the precipitate was removed by centrifugation, and the supernatant was chromatographed by HPLC and the sequence of each purified fragment was analyzed.

Achromobacter lysyl endopeptidase digestion. BANP $(1.5 \mathrm{mg}$ ) was dissolved in $200 \mu \mathrm{l}$ of $10 \mathrm{~mm}$ Tris- $\mathrm{HCl}$ buffer (pH 7.6) with $2 \mathrm{M}$ urea and digested with lysyl endopeptidase for $5 \mathrm{hr}$ at $30^{\circ} \mathrm{C}$. The enzyme to substrate ratio was $1: 300(w t / w t)$. The digest was separated and purified by HPLC.

Pseudomonas fragi endoproteinase Asp-N digestion. BANP, $2.5 \mathrm{mg}$, was dissolved in $300 \mu \mathrm{l}$ of $0.1 \mathrm{M}$ ammonium bicarbonate (pH 8.0 ) and digested with endoproteinase Asp-N for $4 \mathrm{hr}$ at $37^{\circ} \mathrm{C}$. The ratio of enzyme to substrate was $1: 6000(\mathrm{wt} / \mathrm{wt})$. Urea, $72 \mathrm{mg}$, was added to the reaction mixture to dissolve some insoluble materials. Fragments were separated by HPLC.

Trypsin digestion. The intact enzyme or succinylated enzyme, $\left.{ }^{18}\right) 2.0 \mathrm{mg}$, was digested with TPCK-treated tryp$\sin$ at an enzyme-substrate ratio of $1: 50(\mathrm{wt} / \mathrm{wt})$ in $100 \mu \mathrm{l}$ of $0.1 \mathrm{M}$ phosphate buffer, $\mathrm{pH} 7.9$, at $37^{\circ} \mathrm{C}$ for $24 \mathrm{hr}$. The digest was separated by HPLC.

$\alpha$-Chymotrypsin digestion. BANP $(1.9 \mathrm{mg})$ was dissolved in $300 \mu \mathrm{l}$ of $0.1 \mathrm{M}$ ammonium bicarbonate $(\mathrm{pH} 8.0)$ and digested with $\alpha$-chymotrypsin ( 1 to $300, \mathrm{~mol} / \mathrm{mol}$ ) for $3 \mathrm{hr}$ at $37^{\circ} \mathrm{C}$, and then urea, $72 \mathrm{mg}$, was added to the reaction mixture to completely dissolve insoluble peptides. The digest was separated by HPLC and tryptophan-containing peptides were monitored by following the absorbance at $295 \mathrm{~nm}$. TSK-gel ODS120T was used to purify peptides.

Separation and purification of peptides. Peptide mixtures obtained after chemical or enzymatic cleavage were separated by reverse phase HPLC using the system described above by monitoring absorbances at 214,280 , and/or $295 \mathrm{~nm}$. A Vydac C18 column ( $5 \mu \mathrm{m}, 300 \mathrm{~A}, 4.6 \times 250 \mathrm{~mm})$ proved to be most useful. Some fractions were further purified on the same column with a different gradient system (acetonitrile with $0.06 \% \mathrm{TFA}$ ) or on a Waters $\mu$ Bondasphere CN column $(5 \mu \mathrm{m}, 300 \mathrm{~A}, 3.9 \times 150 \mathrm{~mm}$; Waters, MA, U.S.A.) with the above solvent systems.

Amino acid sequence analysis. All of peptides and intact protein were sequenced by manual Edman degradation. ${ }^{193}$ Phenylthiohydantoin (PTH) amino acids were analyzed and quantified by HPLC as reported previously. ${ }^{19)}$ The amino acid sequence of the carboxyl terminal region of the intact protein was analyzed by periodic monitoring of the amino acids liberated by carboxypeptidase $A$ digestion as 
described by Ikenaka. ${ }^{201}$

Amino acid analysis. The amino acid composition of the intact protein was determined as described previously, ${ }^{12}$ except that the tryptophan content was analyzed by the method of Edelhoch. ${ }^{21}$ The amino acid compositions of fragments resulted from chemical or enzymatic cleavages were analyzed by the phenylthiocarbamyl (PTC) amino acid method, ${ }^{18)}$ after hydrolysis with $6 \mathrm{~N} \mathrm{HCl} \mathrm{containing} \mathrm{a}$ trace of phenol at $150^{\circ} \mathrm{C}$ for $1 \mathrm{hr}$.

\section{Results}

$C N B r$-cleavage of $B A N P$, and amino terminal amino acid sequences of the fragments and intact protein

By CNBr-cleavage of BANP, three frag- ments were obtained, after purification by gel filtration followed by HPLC, and their amino acid sequences were analyzed by manual Edman degradation. As shown in Table I, more than 15 residues from the amino termini of the intact protein and its fragments were clearly sequenced in pmol order. These fragments were deduced to be in the order M-1, M2 , and M-3 from the comparison with sequences of the intact protein and thermolysin. However, the sequences of the carboxyl terminal regions of these fragments remain to be clarified.

Table I. Sequence Analysis of Intact Protein and CNBr Fragments

\begin{tabular}{|c|c|c|c|c|c|c|c|c|}
\hline \multirow{2}{*}{ Edman cycle } & \multicolumn{2}{|c|}{ Intact protein } & \multicolumn{2}{|c|}{$M-1$} & \multicolumn{2}{|c|}{$M-2$} & \multicolumn{2}{|c|}{$M-3$} \\
\hline & Residue & $\begin{array}{c}\text { Yield } \\
(\mathrm{pmol})\end{array}$ & Residue & $\begin{array}{c}\text { Yield } \\
\text { (pmol) }\end{array}$ & Residue & $\begin{array}{c}\text { Yield } \\
\text { (pmol) }\end{array}$ & Residue & $\begin{array}{l}\text { Yield } \\
(\mathrm{pmol})\end{array}$ \\
\hline 1 & Ala & 158 & Ala & 567 & Ile & 1226 & Thr & 445 \\
\hline 2 & Ala & 122 & Ala & 949 & Tyr & 584 & His & 352 \\
\hline 3 & Ala & 160 & Ala & 837 & Gly & 273 & Gly & 350 \\
\hline 4 & Thr & 116 & Thr & 700 & Asp & 595 & Val & 903 \\
\hline 5 & Gly & 88 & Gly & 459 & Gly & 320 & Thr & 156 \\
\hline 6 & Ser & 51 & Ser & 23 & Asp & 383 & Gln & 490 \\
\hline 7 & Gly & 64 & Gly & 523 & Gly & 123 & Glu & $46 \mathrm{I}$ \\
\hline 8 & Thr & 58 & Thr & 56 & Ser & 25 & Thr & 178 \\
\hline 9 & Thr & 70 & Thr & 233 & Phe & 87 & Ala & 372 \\
\hline 10 & Leu & 39 & Leu & 258 & Phe & 109 & Asn & 323 \\
\hline 11 & Lys & 9 & Lys & 149 & Ser & 13 & Leu & 348 \\
\hline 12 & Giy & 34 & Gly & 267 & Pro & 21 & Ile & 232 \\
\hline 13 & Ala & 39 & Ala & 140 & Leu & 23 & Tyr & 218 \\
\hline 14 & Thr & 13 & Thr & 106 & Ser & 8 & Glu & 237 \\
\hline 15 & Val & 42 & Val & 216 & Gly & 15 & Asn & 300 \\
\hline 16 & Pro & 37 & Pro & 129 & $?$ & - & Gln & 410 \\
\hline 17 & Leu & 19 & Leu & 116 & Leu & 24 & Pro & 157 \\
\hline 18 & Asn & 21 & Asn & 225 & Asp & 21 & Gly & 128 \\
\hline 19 & & & Ile & 200 & 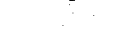 & & $?$ & - \\
\hline 20 & & & Ser & 45 & & & Leu & 161 \\
\hline 21 & & & Tyr & 36 & & & Asn & 128 \\
\hline 22 & & $\cdots$ & Glu & 129 & & & Glu & 292 \\
\hline 23 & & & Gly & 58 & & & $?$ & - \\
\hline 24 & & & Gly & 37 & & & Phe & 44 \\
\hline 25 & & & Lys & 23 & & & Ser & 28 \\
\hline 26 & & & Tyr & 68 & & & Asp & 76 \\
\hline 27 & & & Val & 27 & & & Val & 81 \\
\hline 28 & & & Leu & 13 & & & & \\
\hline 29 & & & $\operatorname{Arg}$ & 12 & & & & \\
\hline $\begin{array}{c}\text { Yield of each } \\
\text { peptide }(\%)\end{array}$ & & & 45 & & 48 & & 11 & \\
\hline
\end{tabular}


Enzymatic digestion of BANP and sequence analyses of the resultant peptides

Figure 1 shows the HPLC profile of the peptides from lysyl endopeptidase digestion. Since BANP has been reported to contain 15 lysyl residues, ${ }^{12\}} 16$ peptides were expected to be produced by lysyl endopeptidase treatment. Unfortunately, however, only 10 peptides were highly purified by HPLC. Some of the peptides appeared insoluble and some were probably not to be eluted from the column under the conditions used. The amino acid sequence of each peptide was established and its location in protein was tentatively assumed to be in the order shown in Table II, from the comparison with sequences of thermolysin and of other Bacillus neutral proteases, most of which were deduced from nucleotide sequencing of the genes. ${ }^{27 \sim 31)}$ About $70 \%$ of the whole sequence were established by these procedures.

Likewise, the intact protein was digested with $P$. fragi endoproteinase Asp-N which is active towards peptide bonds involving the amino groups of asparatyl residues (Fig. 2). Each peptide was purified to homogeneity, and the sequence and organization of each peptide were decided to be as shown in Table III. Surprisingly, the amino terminus of peptide ND-10 was found to be Leu, but the reason remains unknown.

To obtain overlapping peptides, the intact protein and $\mathrm{CNBr}$-fragments were digested with trypsin and $S$. aureus V8 protease (Gluand Asp-specific). Furthermore, the intact protein was succinylated to modify lysyl residues, and then digested with trypsin. By this pro-

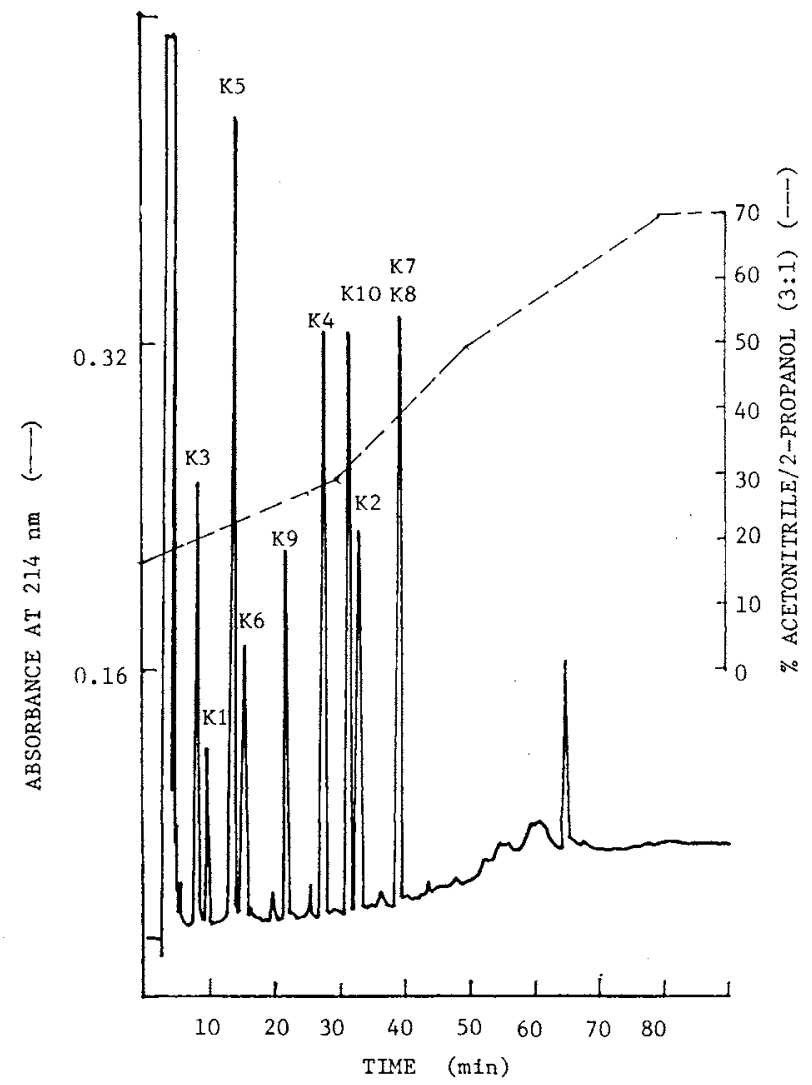

Fig. 1. HPLC Profile of a Lysyl Endopeptidase Digest of BANP.

Lysyl endopeptidase digest was put on a Vydac C18 column as described in Materials and Methods. Fragments $\mathrm{K} 7$ and $\mathrm{K} 8$ were separated by rechromatography using the same column with a different gradient system. The numbering of peptides is based on the sequence order from the amino terminus of the protein. 
Table II. Sequence Analysis of Peptides Obtained by Lysylendopeptidase Digestion

\begin{tabular}{|c|c|c|c|c|c|c|c|c|c|c|}
\hline \multirow{2}{*}{ Edman cycle } & \multicolumn{2}{|c|}{$\mathrm{K}-1$} & \multicolumn{2}{|c|}{$\mathrm{K}-2$} & \multicolumn{2}{|c|}{$\mathrm{K}-3$} & \multicolumn{2}{|c|}{$\mathrm{K}-4$} & \multicolumn{2}{|c|}{$\mathrm{K}-5$} \\
\hline & Residue & $\begin{array}{l}\text { Yield } \\
\text { (pmol) }\end{array}$ & Residue & $\begin{array}{l}\text { Yield } \\
\text { (pmol) }\end{array}$ & Residue & $\begin{array}{c}\text { Yield } \\
(\mathrm{pmol})\end{array}$ & Residue & $\begin{array}{c}\text { Yield } \\
(\mathrm{pmol})\end{array}$ & Residue & $\begin{array}{l}\text { Yield } \\
\text { (pmol) }\end{array}$ \\
\hline 1 & Tyr & 396 & Pro & 482 & Tyr & 511 & Val & 291 & Tyr & 864 \\
\hline 2 & $\mathrm{Val}$ & 270 & Thr & 90 & Phe & 356 & Tyr & 798 & Asn & 658 \\
\hline 3 & Leu & 177 & Gly & 347 & Thr & 322 & Asp & 934 & Gln & 697 \\
\hline 4 & Arg & 201 & Thr & 117 & Ser & 214 & $\mathrm{Tyr}$ & 429 & Pro & 500 \\
\hline 5 & Asp & 354 & Gln & 157 & Ser & 152 & Phe & 247 & Asp & 663 \\
\hline 6 & Leu & 80 & Ile & 145 & Ser & 293 & Tyr & 312 & Asn & 635 \\
\hline 7 & Ser & 117 & Ile & 226 & Gln & 144 & Ser & 113 & Tyr & 377 \\
\hline 8 & Lys & 32 & Thr & 63 & Arg & 42 & Asn & 181 & Ala & 315 \\
\hline 9 & & & Tyr & 197 & Ala & 153 & Phe & 63 & Asn & 91 \\
\hline 10 & & & Asp & 176 & Ala & 126 & & & Tyr & 129 \\
\hline 11 & & & Leu & 97 & $\mathrm{Val}$ & 162 & & & Arg & 173 \\
\hline 12 & & & GIn & 49 & Asp & 184 & & & Asn & 132 \\
\hline 13 & & & Asn & 51 & Ala & 126 & & & Leu & 42 \\
\hline 14 & & & Arg & 18 & $\mathrm{His}$ & 59 & & & Pro & 57 \\
\hline 15 & & & GIn & 20 & Tyr & $3 I$ & & & Asn & 44 \\
\hline 16 & & & & & Asn & 117 & & & & \\
\hline $\begin{array}{c}\text { Yield of each } \\
\text { peptide }(\%)\end{array}$ & 25 & & 48 & & 20 & & 21 & & 47 & \\
\hline
\end{tabular}

Table II. (continued).

\begin{tabular}{|c|c|c|c|c|c|c|c|c|c|c|}
\hline \multirow{2}{*}{ Edman cycle } & \multicolumn{2}{|c|}{$\mathrm{K}-6$} & \multicolumn{2}{|c|}{$\mathrm{K} \cdot 7$} & \multicolumn{2}{|c|}{$\mathrm{K}-8$} & \multicolumn{2}{|c|}{ K-9 } & \multicolumn{2}{|c|}{$K-10$} \\
\hline & Residue & $\begin{array}{c}\text { Yield } \\
\text { (pmol) }\end{array}$ & Residue & $\begin{array}{c}\text { Yield } \\
(\mathrm{pmol})\end{array}$ & Residue & $\begin{array}{l}\text { Yield } \\
(\mathrm{pmol})\end{array}$ & Residue & $\begin{array}{c}\text { Yield } \\
(\mathrm{pmol})\end{array}$ & Residue & $\begin{array}{l}\text { Yield } \\
\text { (pmol) }\end{array}$ \\
\hline 1 & Ala & 196 & Leu & 100 & Ser & 248 & Ala & 5656 & Val & 810 \\
\hline 2 & Ala & 70 & Gly & 125 & $G \ln$ & 433 & Ala & 3014 & Glu & 2342 \\
\hline 3 & Tyr & 114 & Val & 41 & $\mathrm{Gln}$ & 510 & Leu & 4000 & Ala & 958 \\
\hline 4 & Asn & 118 & Ser & 59 & Ile & 103 & Ile & 2890 & Ala & 558 \\
\hline 5 & Thr & 68 & Lys & - & Tyr & 62 & Gin & 5008 & Trp & 195 \\
\hline 6 & Ile & 26 & Ser & 51 & Tyr & 296 & Ser & 946 & Asn & 287 \\
\hline 7 & Thr & 72 & Gin & 53 & Arg & 69 & $\mathrm{Ala}$ & 3237 & Ala & 112 \\
\hline 8 & & & Gin & 61 & Ala & 242 & Arg & 5015 & Val & 59 \\
\hline 9 & & & Ile & 26 & Leu & 71 & Asp & 5105 & Gly & 128 \\
\hline 10 & & & Tyr & 18 & Thr & 117 & Leu & 1290 & Leu & 70 \\
\hline 11 & & & & & Thr & 161 & Tyr & 1039 & & \\
\hline 12 & & & & & Tyr & 99 & Gly & 917 & & \\
\hline 13 & & & & & Leu & 52 & Ser & 507 & & \\
\hline 14 & & & & & Thr & 83 & Thr & 600 & & \\
\hline 15 & & & & & Pro & 161 & Asp & 1229 & & \\
\hline 16 & & & & & Ser & 51 & Ala & 279 & & \\
\hline 17 & & & & & Ser & 42 & Ala & 465 & & \\
\hline 18 & & & & & & & Lys & 100 & & \\
\hline $\begin{array}{c}\text { Yield of each } \\
\text { peptide }(\%)\end{array}$ & 11 & & 11 & & 20 & & .33 & & 52 & \\
\hline
\end{tabular}




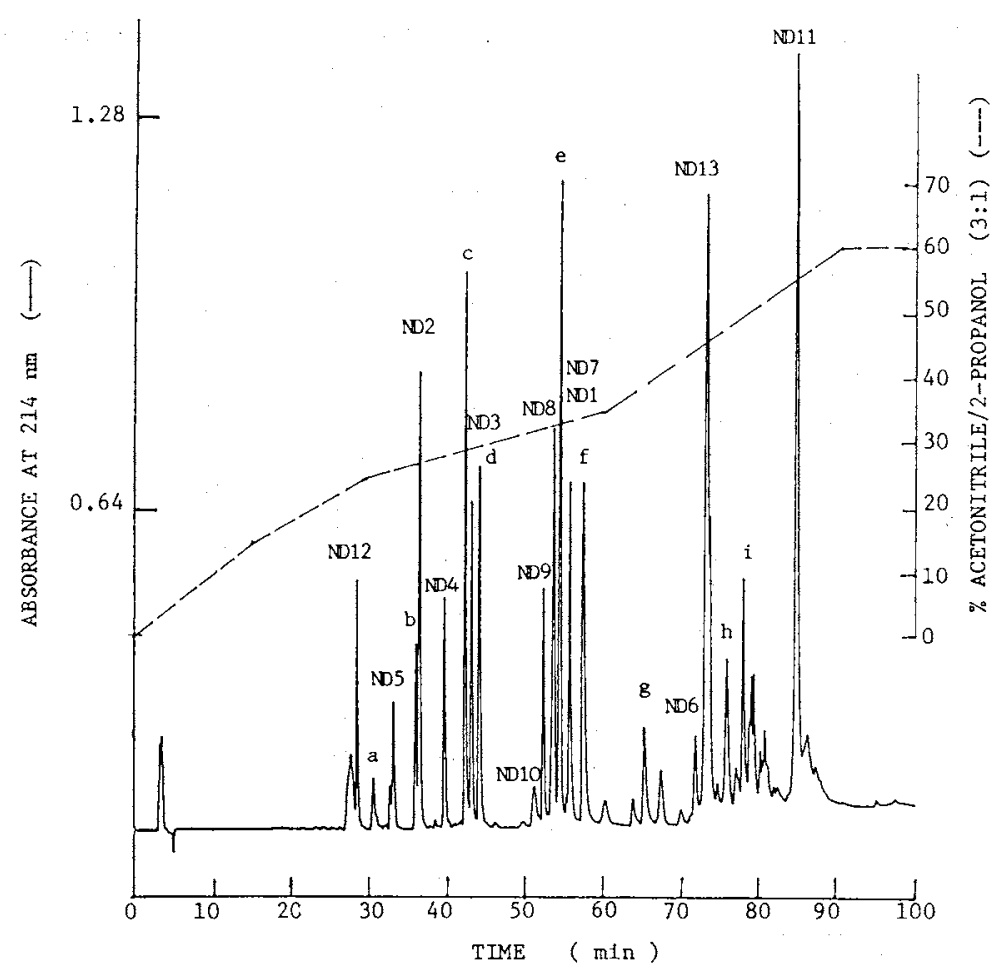

Fig. 2. HPLC Profile of an Endoproteinase Asp-N Digest of BANP.*

The digest was separated by a Vydac C18 column, and some peptides were further rechromatographed using the same column with a different gradient system before sequence analysis.

*The sequences of peaks designated by small letters (a to i) were not shown in Table III, since they were found to be the peptides having the sequences already shown in Tables I and II, after several cycles of manual Edman degradation.

cedure, only the peptide bonds involving arginyl residues became susceptible to trypsin. $\alpha$ Chymotrypsin was also used to get overlapping peptide fragments.

The resultant peptides were separated and purified by HPLC and their amino acid sequences were analyzed. Through these experiments, fragments of unidentified regions and overlapping peptides were obtained (Fig. 3), except for the overlapping peptide linked between the Lys-269 and Asp-270 region. The amino acid sequence of the carboxyl terminus of the intact protein was -Asn-Ala-Val-GlyLeuOH. From these experimental results, the entire amino acid sequence of BANP was deduced to be as shown in Fig. 3.

BANP consists of 300 amino acid residues with a molecular weight of 32,633 . The amino acid composition calculated from sequence analysis and that found by amino acid analysis agreed well, except for serine and isoleucine residues (Table IV).

In Fig. 4, the amino acid sequences of Bacillus neutral proteases hitherto reported are compared with that of BANP. BANP was found to be identical to the neutral protease from $B$. subtilis IA $72,{ }^{24)}$ which was derived from Bacillus subtilis 168.

\section{Discussion}

There are several reports on the sequence analyses of $B$. subtilis neutral proteases, but the entire amino acid sequence was all deduced only from nucleotide sequencing, i.e., from gene analyses of $B$. amyloliquefaciens, ${ }^{22,}{ }^{23)} B$. 
Table III. Sequence Analysis of Peptides Obtained by Endoproteinase ASP-N Digestion

\begin{tabular}{|c|c|c|c|c|c|c|c|c|c|c|c|c|c|c|}
\hline \multirow{2}{*}{$\begin{array}{c}\text { Edman } \\
\text { cycle }\end{array}$} & \multicolumn{2}{|c|}{ ND-1 } & \multicolumn{2}{|c|}{ ND-2 } & \multicolumn{2}{|c|}{ ND-3 } & \multicolumn{2}{|c|}{ ND-4 } & \multicolumn{2}{|c|}{ ND-5 } & \multicolumn{2}{|c|}{ ND-6 } & \multicolumn{2}{|c|}{ ND-7 } \\
\hline & Residue & $\begin{array}{c}\text { Yield } \\
(\mathrm{pmol})\end{array}$ & Residue & $\begin{array}{c}\text { Yield } \\
\text { (pmol) }\end{array}$ & Residue & $\begin{array}{c}\text { Yield } \\
\text { (pmol) }\end{array}$ & Residue & $\begin{array}{c}\text { Yield } \\
\text { (pmol) }\end{array}$ & Residue & $\begin{array}{c}\text { Yield } \\
(\mathrm{pmol})\end{array}$ & Residue & $\begin{array}{c}\text { Yield } \\
\text { (pmol) }\end{array}$ & Residue & $\begin{array}{c}\text { Yield } \\
(\mathrm{pmol})\end{array}$ \\
\hline 1 & Asp & 131 & Asp & 570 & Asp & 455 & Asp & 1307 & Asp & 222 & Asp & 74 & Asp & 1600 \\
\hline 2 & Leu & 13 & Ala & 502 & Tyr & 1325 & Asn & 461 & Gln & 187 & Gly & 197 & $\mathrm{Val}$ & 284 \\
\hline 3 & Gln & 87 & His & 72 & Phe & 457 & Lys & 30 & Met & 158 & Asp & 152 & Thr & 60 \\
\hline 4 & Asn & 50 & Tyr & 460 & Tyr & 909 & Gly & 244 & Ile & 48 & Gly & 80 & Ala & 65 \\
\hline 5 & Arg & 14 & Asn & 273 & Ser & 144 & Ser & 51 & Tyr & 187 & Ser & 62 & $\mathrm{His}$ & 267 \\
\hline 6 & Gin & 36 & Leu & 165 & Asn & 239 & Lys & 23 & Gly & 124 & Phe & 16 & Glu & 146 \\
\hline 7 & Ser & 2 & Gly & 80 & Phe & 76 & Ile & 10 & & & Phe & 16 & Met & 10 \\
\hline 8 & Arg & 9 & Lys & 10 & Lys & 15 & $\mathrm{Val}$ & 90 & & & Ser & 17 & Thr & 20 \\
\hline 9 & $?$ & - & $\mathrm{VaI}$ & 130 & Arg & 42 & Ser & 32 & & & Pro & . 14 & His & 108 \\
\hline 10 & Pro & 18 & Tyr & 148 & Asn & 35 & Ser & 4 & & & Leu & 10 & Gly & 40 \\
\hline 11 & Gly & 6 & & & Ser & 6 & $\mathrm{Val}$ & 27 & & & Ser & 14 & $\mathrm{Val}$ & 5 \\
\hline 12 & Thr & 3 & & & Tyr & 62 & His & 66 & & & Gly & 8 & Thr & 24 \\
\hline 13 & & & & & & & Tyr & 26 & & & Ser & 14 & Gin & 29 \\
\hline 14 & & & & & & & & & & & Leu & 3 & Glu & 10 \\
\hline 15 & & & & & & & & & & & & & $?$ & - \\
\hline 16 & & & & & & & & & & & & & Ala & 13 \\
\hline 17 & & & & & & & & & & & & & Asn & 29 \\
\hline 18 & & & & & & & & & & & & & Leu & 13 \\
\hline 19 & & & & & & & & & & & & & Ile & 10 \\
\hline 20 & & & & & & & & & & & & & Tyr & 26 \\
\hline 21 & & & & & & & & & & & & & Glu & 16 \\
\hline 22 & & & & & & & & & & & & & Asn & 36 \\
\hline $\begin{array}{l}\text { Yield } \\
\text { peptid }\end{array}$ & $\begin{array}{l}f \text { each } \\
(\%)\end{array}$ & 16 & & 28 & & 20 & & 50 & & 15 & & 24 & & 36 \\
\hline
\end{tabular}


Table III. (continued).

\begin{tabular}{|c|c|c|c|c|c|c|c|c|c|c|c|c|c|}
\hline \multirow{2}{*}{$\begin{array}{l}\text { Edman } \\
\text { cycle }\end{array}$} & \multicolumn{2}{|c|}{ ND-8 } & \multicolumn{2}{|c|}{ ND-9 } & \multicolumn{2}{|c|}{ ND-10 } & \multicolumn{3}{|c|}{ ND-1 1} & \multicolumn{2}{|c|}{ ND-12 } & \multicolumn{2}{|c|}{ ND-13 } \\
\hline & Residue & $\begin{array}{l}\text { Yield } \\
(\mathrm{pmol})\end{array}$ & Residue & $\begin{array}{c}\text { Yield } \\
(\mathrm{pmol})\end{array}$ & Residue & $\begin{array}{c}\text { Yield } \\
\text { (pmol) }\end{array}$ & Residue & $\begin{array}{c}\text { Yield } \\
\text { (pmol) }\end{array}$ & $\begin{array}{l}\text { Yield }^{a} \\
\text { (pmol) }\end{array}$ & Residue & $\begin{array}{l}\text { Yield } \\
\text { (pmol) }\end{array}$ & Residue & $\begin{array}{c}\text { Yield } \\
(\mathrm{pmol})\end{array}$ \\
\hline 1 & Asp & 65 & Asp & 1624 & Leu & 1677 & Asp & 1088 & 507 & Asp & 1544 & Asp & 813 \\
\hline 2 & Val & 503 & Ile & 206 & Ser & 1431 & Tyr & 390 & 738 & Ala & 1591 & Ala & 1779 \\
\hline 3 & Phe & 184 & Gly & 731 & Asn & 3385 & Gly & 304 & 821 & Lys & 1073 & $\mathrm{Ala}$ & 1721 \\
\hline 4 & Gly & 267 & Glu & 1031 & Pro & 4571 & Gly & - & 949 & Ala & 767 & Lys & 138 \\
\hline 5 & Tyt & 47 & Asp & 811 & Thr & 444 & Val & 22 & 432 & Ala & 1926 & Val & 470 \\
\hline 6 & Phe & 54 & Ile & 361 & Lys & 423 & $\mathrm{His}$ & 149 & 120 & Leu & 1277 & Glu & 275 \\
\hline 7 & Asn & 26 & Thr & 483 & Tyr & 1486 & Thr & 28 & 250 & Ile & 1161 & Ala & 851 \\
\hline 8 & & & $\mathrm{Val}$ & 300 & & & Asn & 146 & 335 & Gln & 1158 & Ala & 1165 \\
\hline 9 & & & Ser & 211 & & & Ser & 12 & 158 & Ser & 389 & & \\
\hline 10 & & & Gln & 634 & & & Gly & 37 & 160 & Ala & 530 & & \\
\hline 11 & & & Pro & 830 & & & Ile & 10 & 194 & Arg & 45 & & \\
\hline 12 & & & Ala & 52 & & & Pro & 36 & 129 & & & & \\
\hline 13 & & & Leu & 7 & & & Asn & 75 & 54 & & & & \\
\hline 14 & & & Arg & 24 & & & Lys & 6 & 38 & & & & \\
\hline 15 & & & & & & & Ala & 23 & 60 & & & & \\
\hline 16 & & & & & & & Ala & 14 & & & & & \\
\hline 17 & & & & & & & Tyr & 9 & & & & & \\
\hline 18 & & & & & & & Asn & 30 & & & & & \\
\hline 19 & & & & & & & Thr & 8 & & & & & \\
\hline 20 & & & & & & & Ile & 7 & & & & & \\
\hline 21 & & & & & & & Thr & 5 & & & & & \\
\hline 22 & & & & & & & Lys & 5 & & & & & \\
\hline 23 & & & & & & & Leu & 7 & & & & & \\
\hline 24 & & & & & & & Gly & 6 & & & & & \\
\hline 25 & & & & & & & Val & 7 & & & & & \\
\hline 26 & & & & & & & $?$ & - & & & & & \\
\hline 27 & & & & & & & Lys & 4 & & & & & \\
\hline & & & & & & & $?$ & - & & & & & \\
\hline 28 & & & & & & & Gin & 8 & & & & & \\
\hline $\begin{array}{l}\text { Yield } \\
\text { pepti }\end{array}$ & $\begin{array}{l}f \text { each } \\
(\%)\end{array}$ & 30 & & 56 & & 12 & & 40 & & & 56 & & 58 \\
\hline
\end{tabular}


AAATGSGTTLKGATVPLNISYGGGKYYLRDLSKPTTTOIITYDLONROSRLPGTLVSSTTKTPTSSSORAAVDAHYNLGKVYDYFYSNFKRNSYDNKGSK

Intact

Peptides obtained after

cleavage with

$\mathrm{CNBr}$

V8 protease $\mathrm{pH} 4.0$

Lysylendopeptidase

Endoproteinase Asp-N

V8 protease pH 7.9

Trypsin

Trypsin ( suce)

$\mathrm{CNBr}$

V8 protease pH 4.0

Endoproteinsse Asp-N

V8 protease pH 7.9

Trypsin

a-Chymotrypsin

IV--VHYGTQYNNAAWTGDQMIYGD

$$
\begin{aligned}
& \text { GGKYVLRDLSKPTGTQII - YDLQNRQSR } \\
& \text { YVLRDLSK } \\
& \text { PTGTQIITYDLQNRQ } \\
& \text { DLQNRQSR-PGTL }
\end{aligned}
$$

TFTSSSQRAAVDAHYN VYDYFYSNF

$$
\text { DYFYSNFKRNSY }
$$

LPGTLVSSTTK

LPGTLVSSTT-TFTSSSE

NSYDNK

\section{1}

150

IVSSVHYGTQYNNAATTGDQMIYGDGDGSFFSPLSOSLDVTAHEMTHGVTQETANLIYENQPGALNESFSDVFGYFNDTEDWDIGEDITVSQPALRSLSN

$$
\begin{gathered}
\text { IYGDGDGSFFSPLSG-LD THGVTQETANLIYENQPG-LNE-FSDV } \\
\text { TANLIYEN-PG }
\end{gathered}
$$

IVSSVHY

DQMIYG DVTAHEMTHGVTQE-ANLIYEN

DVFGYFN DIGEDITVSQPALR LSN SFSDV-GYF DWDIGE LSN

GYFNDTEDWDIGE

PTKYYOPDNYANYRNLPNTDEGDYGVHTNSG IPNKAAYNTITKL GVSKSQQIYYRALTTYLTPSSTFKDAKAALI IOSARDL,YGSTDAAKYEAAFNAVGL

V8 protease $\mathrm{pH} 4.0$

Lysylendopeptidase

Endoproteinase Asp-N

V8 protease $\mathrm{pH} 7.9$

YNQPDNYANYRNLPN

PTKY

PT - YN

LGVS-SQRIY DYGGVHTNSGIPNKAAYNTITKLGV-K-Q

NLPNTDEGDYGGVH

AA-NTITK

AALIQSARDLYGSTDAAK

AAWNAVG

VEAAWNAVGL DAKAALIQSAR DAAKVEAA

ALTTYLTPSSTFK

Fig. 3. Overlap Strategy of Peptides and the Entire Amino Acid Sequence of Bacillus subtilis amylosacchariticus Neutral Protease.

The top line in boldface represents the entire sequence of BANP. The one letter codes of amino acids below the entire sequence indicate residues found by manual sequence analysis of peptides obtained by chemical or enzymatic cleavages. 
B. amylosacchariticus B. amylollquefaclens $P$ B. amylollquefaciens $F$ B. thermoproteolyticus B. Stearotheromophilus

B. amylosacchartiticus B. amylollquefaclens $p$ B. thermoproteolyticus B. stearotheromophilus B. cereus

B. anylosacchar1ticus B. amylol lavefaciens $P$ B. amyloliquefaclens B. thermoproteolyt1cus B. stearotheromophilus B. cereus

amylosacchariticus B. amyloliquefactens p - amylollquefaciens $F$ - thermoproteolyticus B. cereus

B. amylosacchar 1ticus amyloliquefaclens B. anyloliquefaciens $F$ B. thermoproteclyticus stearotheromoph1lus B. cereus

B. amy Losaccharticus B. amyloliquefaciens $B$. amyloliquefaclens $F$ B. thermoproteolyticus B. stearatheromophlius B. cereus

B. anylosacchariticus B. amyioliquefaciens $\mathrm{P}$ B. thermaproteolyticus B.
B.
B.
thermaproteolyticus
stearotheromophilus
Sal-

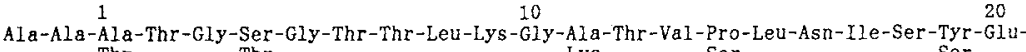
10 -Thr- -Thr-Thr- -Thr-Thr-Ser- -Val-Gly-Val- Arg-Gly-2.---ileVal-Ala -Thr-Asn-Lys-Val-Gly-ThrSer-..-v-Val-

-Leu-GIY-Asp-GIn-Lys-Asn-Ile-Leu-Gly-Asn-Gln-Lys- -Ile-

-Leu-GIy-Asp-Thr-Lys-Ser-Leu-
40

Gly-GIy-Lys-Tyr-Val--.-Ser-

Asn-Thr-Thr- -Ser-.-.....-Thr-Tyr-Tyr-Tyr-

Asn-Thr-Thr- -Ser-Ser-Tyr-Tyr-Gly-Tyr-Tyr-Tyr

$-G I n-A s n-A s n-$

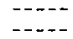

-Arg-

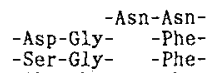

- Ser-Gly- -Phe-
50 60

Thr-Tyr-Asp-Leu-Gln-Asn-Arg-Gln-Ser-Arg-Leu-Pro-Gly-Thr-Leu-Val-Ser-Ser-Thr-Thr-Lys -Glu-Tyr-Asn-Glu-Tyr-Asn-Ala-Lys-Tyr- - - - - Thr-Thr$-A s n$ $\begin{array}{ll}\text {-Gly-Arg- } & ---- \text { Thr-Val- } \\ \text {-Ala-Lys- } & --.- \text { Thr- }\end{array}$
$-\operatorname{Ser}-$ -Ser-
-Ser-Trp-Ala-Asp-Ala-Aso-Asn-Trp-Thr-Asp-Gly-Asp-Asn-Trp-Ala-Asp-Ala-As-Asn80

70
Thr-Phe-Thr-Ser-Ser-Ser-GIn-Arg-Ala-Ala-VaI-Asp-Ala-H1s-Tyr-Asn-Leu-Gly-Lys-VaIGin- $\quad-$ Thr-

Gin- -Phe-Ala- -Tyr-Asp-Ala-Pro

-Ala- -Tyr-Asp-Ala$\begin{array}{lr}\text { Tyr-Ala- } & -\mathrm{Val}- \\ \text {-Tyr-Ala- } & - \text { Thr }\end{array}$
-Tyr-Ala- -VBi-Thr-

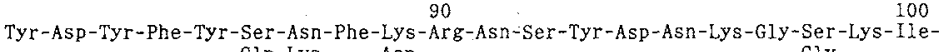
- Glu-Gln-Lys- -Asn-
-GIn-Lys-Tyr-Asn
-Tyr-Lys-Asn-Val-His-Asn-
-Tyr-Lys-Asn-Val-His-Giy-.
-Tyr-Lys-Ala-Thr- - - -Asn$-\mathrm{Gly}-$
$-\mathrm{Gly}-$

- Gly $-A s n-A s n-A l a-A l a-$ Gly-Ser-Asn-AIa-Ala -Ile-Asn-Asp-Ala- -AIa-Pro-Leu-

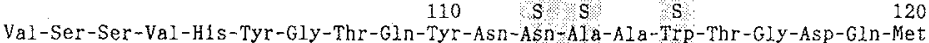

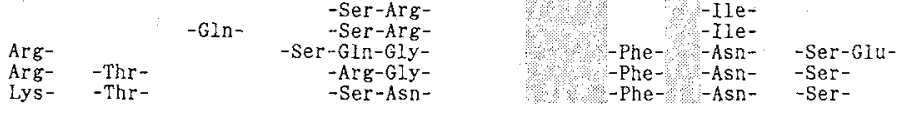
Ile-Tyr-Gly-Asp-Gly-Asp-Gly-Ser-Phe-Phe-Ser-Pro-Leu-Ser-Gly-Ser-Leu-Asp-Val-Thr-

$\begin{array}{ll}\text {-Gin-Thr- } & \text {-Ile- } \\ \text {-Gln-Thr- } & \text {-Leu- -Phe- } \\ \text {-Val-Thr- } & \text {-Thr-Ser- }\end{array}$

$$
- \text { Met- }
$$$$
-G l y-I i e-
$$

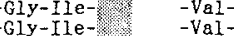

-Gly-Ile- -
c.
160

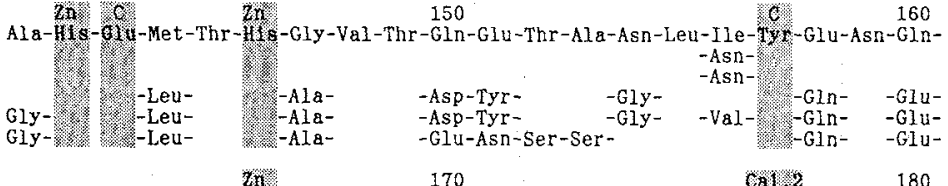

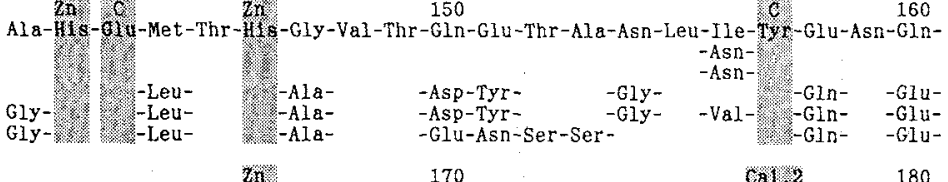

B. amylosacchartticus B. amylollquefoctens $P$ 8. amyloliquefaclens

B. thermoproteolyticus B. stearotheromophilus B. cereus

B. anylosacchariticus $\frac{B}{B}$ amylollquefaclens $P$ B. amyloliquefaclens $F$ B. thermoproteolyticus B. stearotheromophilus B. cereus

B. amylosacchariticus $\frac{B}{B}$. amyloliquef aclens $P$ B. amyloliquefaciens $\mathrm{F}$ $\bar{B}_{\text {. }}$ stearotheromoghilus B. cereus Pro-GIy-Ala-Leu-Asn-Gilit-Ser-Phe-Ser-Asp-Val-Phe-GIy-Tyr-Phe-Asn-Gsp-Thr-GIu---

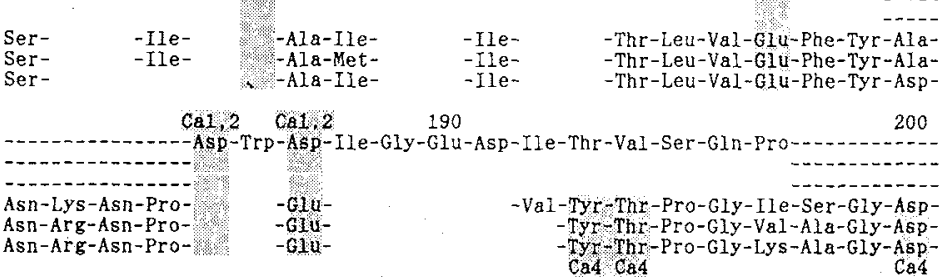

B. amylosacchariticus B. amyloliquefaclens B. amyloliguefaciens B. thermoproteolyticus B. B. . cereus

B. anylosacchariticus B. amyloliquefaclens $P$ $\bar{B}$ anyloliquefaclens $\mathrm{F}$ B. thermoproteolyticus B. stearotheromopht lus B. cereus

Ala-Leu-Ara-Ser-leu-Ser-Asn-Pro-Thr 210

$\begin{array}{llll}\text {-Met- } & \text {-Asp- } & \text {-Ala- } & \text {-GIy- } \\ \text {-Met- } & \text {-Asp- } & \text {-AIz- } & \text {-Gly-Asp- } \\ \text {-Met- } & \text {-Asp- } & & \text {-Gly-Asp- }\end{array}$

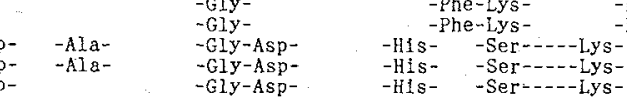

Asn-Leu-Pro-Asn-Thr-Asp-Glu-Gly-Asp-Tyr-Gly-GIy-VaI-GIs-Thr-Asn-Ser-Gly-Ile-Pro-Asn-Lys-Ala-Ala-

Tyr-Thr-Gly-Thr-GlnTyr-Thr-GIy-Thr-GlnTyr-Thr-Gly-Ser-Ser-

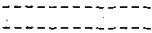

-
-IJe-

-Ile-

- IIe-

$-\mathrm{Gln}-$ 


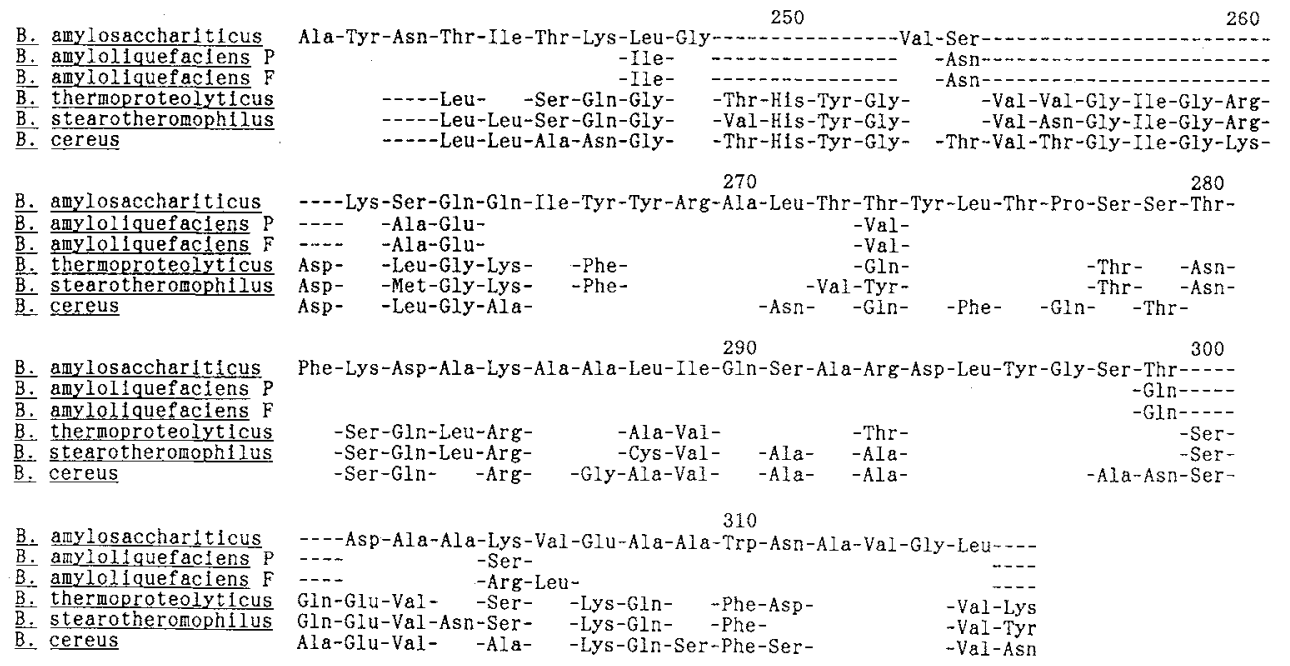

Fig. 4. Sequence Homology Found in Bacillus Neutral Proteases.

The numbering of amino acid residues is based on that for thermolysin. $\mathrm{C}$, residues involved in catalytic reaction; $\mathrm{S}$, residues related to interaction with substrate; $\mathrm{Zn}$, zinc-binding ligands; $\mathrm{Ca}$, residues participating in binding of Ca-1, 2, 3 and 4 in thermolysin.

subtilis GSY264* (B. subtilis IA72), ${ }^{24)}$ and of $B$. amylosacchariticus (Yoshimoto et al., unpublished). On the other hand, protein sequencing was attempted for the neutral protease A of B. amyloliquefaciens, but the results were incomplete. $\left.{ }^{25}\right)$

This paper is the first report on the complete amino acid sequence of a neutral protease from Bacillus subtilis, found from protein sequencing. The amino acid sequence of BANP was identical to that of $B$. subtilis IA72, which was deduced from nucleotide sequencing. ${ }^{24)}$ The enzyme was $89 \%$ homologous to the neutral protease of $B$. amyloliquefaciens $\mathrm{P}$ (ATCC 23844), ${ }^{22)} 87 \%$ homologous to $B$. amyloliquefaciens F(ATCC 23350), ${ }^{23)} 47$ and $48 \%$ homologous to thermolysin ${ }^{6)}$ and the thermostable neutral protease from $B$. stearothermophilus $\mathrm{CU} 21,{ }^{26)}$ and $45 \%$ homologous to $B$. cereus enzyme. ${ }^{27)}$ The putative active site amino acid residues (Glu-143, Tyr157, Asp-226 and His-231), which seem to be involved in catalytic reaction, $\mathrm{Zn}$-binding ligands (His-142, His-146, and Glu-166) and residues related to interaction with the substrate (Asn-112, Ala-113, Trp-115, and Arg-
203) were well conserved among them, as deduced from comparison with thermolysin. ${ }^{7}$ 28, 29)

These results indicate that the reaction mechanisms of Bacillus neutral proteases for peptide bond hydrolysis are quite similar. Of four amino acid residues involved in binding of Ca-1 and Ca-2, Asp-138 and Asp-185 were conserved in Bacillus neutral proteases, but the other two, Glu-177 and Glu-187, in thermolysin were replaced by Asp in B. subtilis neutral proteases. On the other hand, $\mathrm{Ca}$ binding ligands for Ca-3 (Asp-57 and Asp-59) and Ca-4 (Tyr-193, Thr-194 and Asp-200) in thermolysin were not conserved; Asp-57 and Asp-59 for Ca-3 were replaced by Ser and $\mathrm{Thr}$, respectively, and those for $\mathrm{Ca}-4$ were by Thr-193 and Val-194, Asp-200 being deleted in B. subtilis neutral proteases. These discrepancies seem to be related to the instability of B. subtilis neutral proteases towards autodigestion and/or heat treatment, though BANP was supposed to contain 3 or 4 gram atoms of Ca per mol. ${ }^{10,}$, 30)

In the genus Bacillus, cases where an identical protease is produced by different strains are

* Bacillus subtilis GSY $264,{ }^{24)}$ which was derived from $B$. subtilis 168 , is now registrated as $B$. subtilis IA72 at the Bacillus Genetic Stock Center, Ohio State University (comment from Dr. Donald H. Dean). 
Table IV. Amino ACID Composition of B. amylosacchariticus NeUTRAL Protease

\begin{tabular}{|c|c|c|c|}
\hline & \multirow{2}{*}{ Sequencing } & \multicolumn{2}{|c|}{ Amino acid analysis } \\
\hline & & Calcd. & Integral \\
\hline Lysine & 15 & 14.8 & 15 \\
\hline Histidine & 5 & 5.1 & 5 \\
\hline Arginine & 9 & 9.0 & 9 \\
\hline Aspartic acid & 20 & 41.8 & 42 \\
\hline Asparagine & 22 & & \\
\hline Threonine & 30 & $29.8^{a}$ & 30 \\
\hline Serine & 30 & $28.8^{a}$ & 29 \\
\hline Glutamic acid & 9 & 22.4 & 22 \\
\hline Glutamine & 13 & & \\
\hline Proline & 11 & 10.5 & 11 \\
\hline Glycine & 26 & 25.8 & 26 \\
\hline Alanine & 26 & 26.1 & 26 \\
\hline 1/2 Cystine & 0 & 0 & 0 \\
\hline Valine & 15 & $15.4^{b}$ & 15 \\
\hline Methionine & 2 & $2.0^{b}$ & 2 \\
\hline Isoleucine & 12 & $10.6^{b}$ & 11 \\
\hline Leucine & 21 & 21.0 & 21 \\
\hline Tyrosine & 22 & 22.4 & 22 \\
\hline Phenylalanine & 9 & 8.7 & 9 \\
\hline Tryptophan & 3 & $2.6^{c}$ & 3 \\
\hline Total & 300 & & 298 \\
\hline
\end{tabular}

a Values extrapolated to zero hydrolysis time.

$b$ Maximal values were adopted.

c Determined by the method of Edelhoch. ${ }^{21}$

very rare; for example, even in the neutral proteases produced by two strains of the same Bacillus species, B. amyloliquefaciens strains $\mathrm{P}^{22)}$ and $\mathrm{F}^{231}$ there were observed 11 alternation in nucleotides of the genes encoding mature enzyme protein, resulting in 8 amino acid changes out of 314 residues of the mature protein. A quite similar relationship was also observed between subtilisin $\mathrm{AM}^{15)}$ produced by $B$. amylosacchariticus and subtilisin $\mathrm{E}^{31)}$ purified from $B$. subtilis 168 , the parent strain of $B$. subtilis IA72; comparison of nucleotide sequences of both subtilisins indicated that there were 10 discrepancies in nucleotides encoding mature enzymes, which resulted in alternation of two amino acid residues between two subtilisins.

Nevertheless, B. subtilis IA72 and amylosacchariticus were found to produce an identical neutral protease. This was also confirmed by nucleotide sequencing of the gene encoding BANP (Yoshimoto et al., unpublished) and comparison with that encoding $B$. subtilis IA 72 neutral protease. ${ }^{24)} B$. subtilis neutral proteases are commonly unstable, as compared with thermolysin and subtilisins. Taking into account these facts, it might be possible to conclude that $B$. subtilis amylosacchariticus and IA72 are taxonomically very close and presumably be derived from a single ancestor, B. subtilis 168 , and that a minor alteration in the amino acid sequence of the neutral protease make the enzyme more unstable, leading the mutated proteases to be excluded from nature.

Acknowledgment. The authors are grateful to Messrs. A. Kanatani and T. Tsuji for their assistance in this experiment.

\section{References}

1) D. Tsuru and T. Yoshimoto, "Microbial proteases," in Handbook of Microbiology, Vol. VIII, ed. by A. I. Laskin and H. A. Lechevalier, CRC Press Inc., Florida, 1987, pp.239 283.

2) F.S. Markland, Jr. and E. L. Smith, "The Enzymes," 3rd Ed., Vol. 3, Academic Press, New York, 1971, p. 561.

3) M. Ottesen and I. Svendsen, Methods Enzymol., 19, $199(1970)$.

4) C. S. Wright, R. A. Alden and J. Kraut, Nature, 221, 235 (1969)

5) H. Matsubara and J. Feder, "The Enzymes," 3rd Ed., Vol. 3, Academic Press, New York, 1971, p. 721.

6) K. Titani, M. A. Hermodson, L. H. Ericsson, K. A. Walsh and H. Neurath, Nature, New Biol., 238, 35 (1972).

7) P. M. Colman, J. N. Jansonius and B. W. Matthews, J. Mol. Biol., 70, 701 (1972).

8) J. Fukumoto, T. Yamamoto and K. Ichikawa, Proc. Japan Acad., 27, 352 (1951).

9) J. Fukumoto, Nippon Nögeikagaku Kaishi, 17, 487 (1943).

10) D. Tsuru, T. Yamamoto and J. Fukumoto, Agric. Biol. Chem., 30, 651 (1966); ibid, 30, 856 (1966).

11) D. Tsuru, H. Kira, T. Yamamoto and J. Fukumoto, Agric. Biol. Chem., 30, 1261 (1966); ibid., 31, 330 (1967).

12) D. Tsuru, T. Yoshimoto, T. Yoshida, H. Kira and J Fukumoto, Int. J. Protein Res., 2, 75 (1970).

13) D. Tsuru, T. Yoshida, T. Hirose, T. Yoshimoto and J. Fukumoto, Int. J. Protein Res., 2, 257 (1970). 
14) M. Kurihara, F. S. Markland and E. L. Smith, J. Biol. Chem., 247, 5619 (1972).

15) T. Yoshimoto, H. Oyama, T. Honda, H. Tone, T. Takeshita, T. Kamiyama and D. Tsuru, J. Biochem., 103, 1060 (1988).

16) J. D. McConn, D. Tsuru and K. T. Yasunobu, $J$. Biol. Chem., 239, 3706 (1964).

17) D. Tsuru, J. D. McConn and K. T. Yasunobu, J. Biol. Chem., 240, 2415 (1965).

18) G. E. Tarr, in "Methods of Protein Microcharacterization," ed. by J. E. Shively, Humana Press, Clifton, N.J., 1986, pp. 155 194.

19) R. Kobayashi and G. E. Tarr, Proteins, Nucleic Acids and Enzymes, 31, 991 (1986).

20) T. Ikenaka, in "Chemistry of Proteins" (in Japanese), ed. by K. Narita and T. Murachi, Tokyo Kagaku Dojin, Tokyo, 1976, pp. $203 \sim 211$.

21) H. Edelhoch, Biochemistry, 6, 1948 (1967).

22) N. Vasantha, L. D. Thompson, C. Rhodes, C. Banner, J. Nagle and D. Filpula, J. Bacteriol., 159, 811 (1984).
23) H. Shimada, M. Honjo, I. Mita, A. Nakayama, A. Akaoka, K. Manabe and Y. Furutani, J. Biotechnol., 2, 75 (1985).

24) M. Y. Yang, E. Ferrari and D. J. Henner, $J$. Bacteriol., 160, 15 (1984).

25) P. L. Levy, M. K. Pangburn, Y. Burstein, L. H. Ericsson, H. Neurath and K. A. Walsh, Proc. Natl. Acad. Sci., U.S.A., 72, 4341 (1975).

26) M. Takagi, T. Imanaka and S. Aiba, J. Bacteriol., 163, 824 (1985).

27) W. Silder, E. Niederer, F. Suter and H. Huber, Biol. Chem. Hoppe-Seyler, 367, 643 (1986).

28) A. F. Monzingo and B. W. Matthews, Biochemistry, 23, 5724 (1984).

29) H. M. Holden, D. E. Tronrud, A. F. Monzingo, L. H. Weaver and B. W. Matthews, Biochemistry, 26, 8542 (1987).

30) M. Tajima, I. Urabe, K. Yutani and K. Okada, Eur. J. Biochem., 64, 243 (1976).

31) M. L. Stahl and E. Ferrari, J. Bacteriol., 158, 411 (1984). 